\title{
Study of Hematological Parameters and Bone Marrow in Pancytopenia
}

\author{
${ }^{1}$ Shreyanshu Sahay, ${ }^{2}$ Ramesh ST
}

\section{ABSTRACT}

Background: Pancytopenia is a striking feature of much serious and life-threatening illness. The etiological diagnosis is essential for the clinical management and prognosis of the patient. Routine hematological investigations, examination of the bone marrow by aspiration and biopsy provides various details that help in understanding the pathogenesis of pancytopenia.

Objective: To diagnose and know the etiology of pancytopenia through hematological evaluation and bone marrow examination.

Setting and design: This is a cross-sectional study done in Department of Pathology, Sri Siddhartha Medical College and Hospital, Tumkur for a period of 18 months from 2016 to 2018.

Material and methods: Patients belonging to all age groups found to have pancytopenia on complete blood count were included in the study. However, those who were on chemotherapy treatment were excluded. A total of 85 cases were included in our study.

Primary hematological procedures and bone marrow aspiration were performed in all the patients, and trephine biopsy was done in 2 cases. Statistical data of age, sex, chief complaints, physical findings, peripheral blood examination, bone marrow aspiration findings and trephine biopsy in 2 were studied.

Observations and results: Out of 85 patients, the most common cause of pancytopenia in our study was combined deficiency anemia (megaloblastic anemia and iron deficiency anemia) seen in 44 cases. Other causes were megaloblastic anemia seen in 27 cases, myelodysplastic syndrome seen in 7 cases, iron deficiency anemia seen in 3 cases, normal bone marrow in 3 cases and 1 case of malaria.

Conclusion: Pancytopenia is a life-threatening illness. The physical findings and complete blood count provide valuable information in the patients with pancytopenia. Bone marrow study (aspiration/ biopsy) provides confirmation of the diagnosis.

Keywords: Bone marrow aspiration, Combined deficiency anemia, Megaloblastic anemia, Pancytopenia.

How to cite this article: Sahay S, Ramesh ST. Study of Hematological Parameters and Bone Marrow in Pancytopenia. J Med Sci 2018;4(4):111-114.

\footnotetext{
${ }^{1}$ Postgraduate Student, ${ }^{2}$ Professor

1,2Department of Pathology, Sri Siddhartha Medical College and Research Centre, Tumkur, Karnataka, India

Corresponding Author: Shreyanshu Sahay, Postgraduate Student, Department of Pathology, Sri Siddhartha Medical College and Research Centre, Tumkur, Karnataka, India, email: shreyanshusahay88@gmail.com
}

\section{Source of support: Nil}

Conflict of interest: None

\section{INTRODUCTION}

Pancytopenia is not a disease entity but a triad of findings that may result from a number of disease processes and is defined as the simultaneous presence of anemia, leucopenia, and thrombocytopenia. Bone marrow aspiration along with trephine biopsy is important to find out the cause of pancytopenia.

Peripheral pancytopenia is usually a manifestation of bone marrow disorders either primary or secondary. The presenting symptoms are usually attributable to anemia, leucopenia or thrombocytopenia, which in later stages is responsible for the downhill course.

Physical findings and peripheral blood picture provide valuable information in the workup of pancytopenic patients and help in planning investigations on bone marrow samples.

In addition to the routine hematological investigations, examination of the bone marrow by aspiration and biopsy provides additional details that help in understanding the pathogenesis behind pancytopenia in a particular case. The etiology of pancytopenia can thus be established after correlation of the clinical details with the laboratory investigations. ${ }^{2}$

The causes of pancytopenia can be (a) ineffective hematopoiesis with cell death in the marrow, (b) formation of defective cells which are rapidly removed from circulation, (c) sequestration and/or destruction of cells by the action of antibodies or, (d) trapping of normal cells in a hypertrophied and over-reactive reticuloendothelial system. $^{3}$

The pattern of diseases leading to pancytopenia is expected to vary in different population groups with their difference in age pattern, nutritional status, and prevalence of infective disorders.

The severity of pancytopenia and the underlying pathology determines the management and prognosis of these patients. The etiology of pancytopenia can vary from treatable disorders-such as megaloblastic anemia to more serious conditions such as the myelodysplastic syndromes which increase the likelihood of developing hematological malignancies in future. ${ }^{2}$ 


\section{MATERIALS AND METHODS}

The present study was carried out on 85 patients in the Department of Pathology, Sree Siddhartha Medical College and Research Centre, Tumkur, Karnataka, India for 18 months from 2016 to 2018. A detailed clinical history, general and systemic examination was done for all the patients. ${ }^{2} \mathrm{~mL}$ of EDTA anticoagulated blood was collected and processed through Sysmex XP100-A1428, and all the hematological parameters were obtained. Smears were prepared and stained with Leishman stain for reporting. Bone marrow aspiration was done for all the patients under local anesthesia.

\section{RESULTS}

A total number of 85 patients with pancytopenia were studied. The age of the patients ranged from 15 to 83 years with a mean age of 42.36 years. Both males and females were almost equally affected. Male to female ratio was 1.02:1.

The most common mode of presentation was a generalized weakness, which was present in 79 (92.9\%) patients. The other main symptoms were dyspnea, fever, giddiness, palpitation, and fatigability. Pallor was a common finding in all the patients; splenomegaly and hepatomegaly were seen in $23.5 \%$ and $21.1 \%$ patients, respectively. Other physical findings were edema, jaundice, skin changes, clubbing, and ascites.

In the present study, the commonest cause of pancytopenia was combined/ dual deficiency anemia which constituted $51.8 \%$ of the total, followed by megaloblastic anemia in $31.8 \%$ cases. About $8.2 \%$ of cases of myelodysplastic syndrome, $3.5 \%$ of cases of iron deficiency anemia and $3.5 \%$ of normal bone marrow were seen. A single case of malaria constituting $1.2 \%$ total was also detected (Table 1).

A detailed peripheral smear examination was done on all the patients. Anisopoikilocytosis was a significant finding ( $p$ value $=0.001)$ in combined deficiency anemia and megaloblastic anemia. Majority of the combined deficiency anemia had dimorphic blood picture (Fig. 1A). Megaloblastic anemia had macrocytic RBCs in most of the case followed by dimorphic RBCs. Myelodysplastic

Table 1: Causes of pancytopenia in 85 patients

\begin{tabular}{lll}
\hline Causes & Frequency & Percent \\
\hline Combined deficiency & 44 & 51.8 \\
Megaloblastic anemia & 27 & 31.8 \\
Myelodysplastic syndrome & 7 & 8.2 \\
Iron deficiency anemia & 3 & 3.5 \\
Normal bone marrow & 3 & 3.5 \\
Malaria & 1 & 1.2 \\
\hline Total & 85 & 100.0 \\
\hline
\end{tabular}

syndrome cases showed normocytic normochromic followed by dimorphic RBC morphology. Cases with normal bone marrow and malaria have normocytic normochromic RBCs.

Bone marrow aspiration was performed on all the 85 patients. Hyper-and hypo-cellular marrow was observed in 3 and 7 cases, respectively (Fig. 1B). Rest all the cases had normocellular marrow. Erythroid hyperplasia was the significant finding ( $p$ value $=0.0005)$ in combined deficiency anemia followed by megaloblastic anemia and myelodysplastic syndrome (Fig. 1C). Dysgranulopoiesis including giant metamyelocytes was observed in combined deficiency anemia, megaloblastic anemia, myelodysplastic syndrome as well as in one case of iron deficiency anemia. Dysmega-karyopoiesis including hypolobated and hyperlobated forms were observed in 13 cases of combined deficiency anemia followed by megaloblastic anemia and myelodysplastic syndrome and one case of malaria and iron deficiency anemia. Malarial parasites were observed in one case.

Trephine biopsy was performed in 2 cases. One showed feature of micronormoblastic megaloblastic bone marrow with erythroid hyperplasia and was diagnosed as combined deficiency anemia (Fig. 1D). Other case showed the features of megaloblastic bone marrow.

\section{DISCUSSION}

Pancytopenia is one of the common hematological findings with variable clinical presentations.
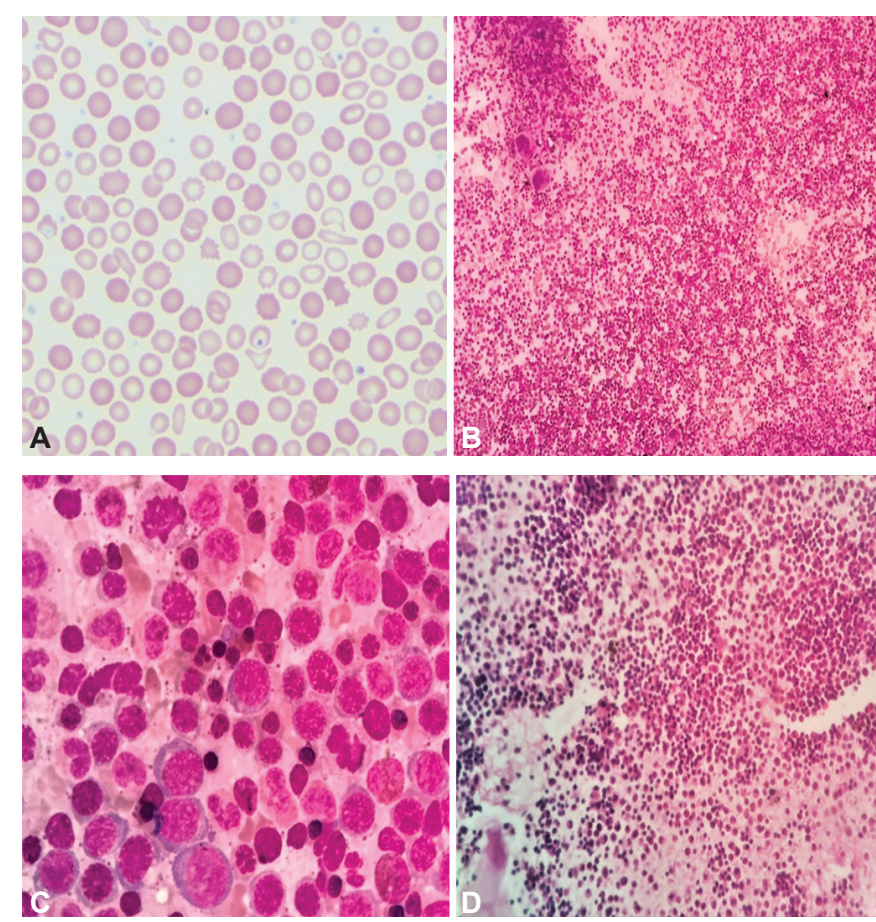

Figs 1A to D: (A) Dimorphic blood picture (Leishman stain, 10x); (B) Hyper cellular marrow (Leishman stain, 10x); (C) Combined deficiency anemia (Leishman stain, 100x); (D) Bone marrow biopsy-combined deficiency anemia 
In the present study, the most common cause of pancytopenia was combined deficiency/nutritional anemia (megalo-blastic anemia with iron deficiency anemia) seen in $51.8 \%$ cases followed by megaloblastic anemia seen in $31.8 \%$ cases. These were in concordance the study conducted by Basha et al. where combined deficiency was the most common cause of pancytopenia with $30 \%$ cases followed by megaloblastic anemia seen in $28 \%$ cases. $^{2}$ However, in other studies conducted by Dagdia et al. and Javalgi et al. megaloblastic anemia was the most common cause for pancytopenia seen in $29.3 \%$ and $72.6 \%$ cases, respectively (Table 2). ${ }^{4,5}$ The high incidence of combined deficiency anemia in our study can be attributed to the high prevalence of vitamin and iron deficiencies in our country. ${ }^{2}$ The patients in our study belonged to the lower socioeconomic strata, putting them at higher risk of severe nutritional deficiencies.

The myelodysplastic syndrome was observed in $8.2 \%$ cases in our study which was similar to the studies conducted by Dagdia et al. and Pathak et al. where $8 \%$ and $5.8 \%$ cases of the myelodysplastic syndrome were observed, respectively. ${ }^{4,6}$ Bone marrow aspirate examination helped to confirm the diagnosis.

Other causes of pancytopenia observed in our study were iron deficiency anemia seen in 3.5\% cases which were in concordance with Javalgi et al. and Desalphine et al. where $12.2 \%$ and $2 \%$ cases of iron deficiency anemia were seen, respectively. ${ }^{5,7}$ This reflects the higher prevalence of nutritional anemia in Indian subjects. Iron deficiency is generally associated with a reactive thrombocytosis, however, increasing severity of iron deficiency leads to normalization and occasionally even decrease platelet counts. The exact mechanism of this is unclear but may be related to the alteration in the activity of iron-dependent enzymes in thrombopoiesis and leucopoiesis. ${ }^{5}$

About $3.5 \%$ of the patients also showed normal bone marrow finding in our study which was similar to the study conducted by Pathak et al. and Desalphine et al. where normoblastic bone marrow was seen in $5.8 \%$ and $22 \%$ of all the cases respectively. ${ }^{6,7}$ The patients were diagnosed with viremia in all the 3 cases resulting in pancytopenia on the peripheral blood picture.

A single case of malaria constituting $1.2 \%$ of all the cases was seen in our study, which was in concordance with the study conducted by Javalgi et al. where $3.7 \%$ cases of malaria were seen. ${ }^{5}$ Tumkur is one of the 13 malaria endemic districts of Karnataka. On average, $>15,000$ malaria cases are reported annually with $>25 \%$ of P. falciparum cases. ${ }^{8}$ In our study, the diagnosis was confirmed by demonstrating gametocyte of $P$. falciparum in the bone marrow aspirate.

In the present study, detailed peripheral smear examination and bone marrow study were done for all the cases. Anisopoikilocytosis, NRBCs, and polychromatophils were significant findings in cases combined deficiency anemia. Twenty-four $(28.2 \%)$ out of $44(51.8 \%)$ cases showed abnormal leukocytes in the in the form of hypersegmented neutrophils and relative lymphocytosis in $12(27.3 \%)$ cases each. Dimorphic blood picture was the most common type of Rbc morphology accounting for $41.1 \%$ of the total cases. This was in concordance with the study conducted by Basha et al. ${ }^{2}$ and Desalphine et al. ${ }^{7}$ Bone marrow examination helped to identify and confirm the underlying pathology in all the cases. All the cases of combined deficiency anemia showed micronormoblastic megaloblastic bone marrow. Thirty-two $(72.7 \%)$ out of 44 cases showed erythroid hyperplasia.

Table 2: Various causes of pancytopenia compared to other studies of pancytopenia:

\begin{tabular}{|c|c|c|c|c|c|c|}
\hline Causes & Basha et al. $^{2}$ & Dagdia et al. ${ }^{4}$ & Pathak et al. ${ }^{6}$ & Javalgi et al. ${ }^{5}$ & Desalphine et al. ${ }^{7}$ & Present study \\
\hline Combined deficiency & $15(30 \%)$ & $9(12 \%)$ & - & - & $4(8 \%)$ & $44(51.8 \%)$ \\
\hline Megaloblastic anemia & $14(28 \%)$ & $22(29.3 \%)$ & $12(11.7 \%)$ & $77(72.6 \%)$ & $8(16 \%)$ & $27(31.8 \%)$ \\
\hline Myelodysplastic syndrome & $2(4 \%)$ & $6(8 \%)$ & $6(5.8 \%)$ & $1(0.9 \%)$ & - & $7(8.2 \%)$ \\
\hline Iron deficiency anemia & - & - & - & $13(12.2 \%)$ & $1(2 \%)$ & $3(3.5 \%)$ \\
\hline Normal bone marrow & - & - & $6(5.8 \%)$ & - & $11(22 \%)$ & $3(3.5 \%)$ \\
\hline Malaria & - & - & - & $4(3.7 \%)$ & - & $1(1.2 \%)$ \\
\hline Aplastic anemia & $5(10 \%)$ & $14(18.6 \%)$ & $33(32.3 \%)$ & $2(1.8 \%)$ & $13(26 \%)$ & - \\
\hline Leukemia/lymphoma & $2(4 \%)$ & $13(17.3 \%)$ & $11(10.8 \%)$ & $3(2.8 \%)$ & $9(18 \%)$ & - \\
\hline Hypersplenism & $3(6 \%)$ & $6(8 \%)$ & - & $1(0.9 \%)$ & - & - \\
\hline Myelofibrosis & - & - & - & $1(0.9 \%)$ & $4(8 \%)$ & - \\
\hline Storage disease & - & - & $1(0.9 \%)$ & - & - & - \\
\hline Multiple myeloma & - & $1(1.3 \%)$ & $2(1.9 \%)$ & $1(0.9 \%)$ & - & - \\
\hline $\begin{array}{l}\text { Metastasis to bone } \\
\text { marrow }\end{array}$ & - & $1(1.3 \%)$ & - & - & - & - \\
\hline SLE & - & - & - & $3(2.8 \%)$ & - & - \\
\hline
\end{tabular}




\section{CONCLUSION}

The present study shows that physical findings, complete blood count and peripheral blood smear examination provide valuable information in the workup of pancytopenic patients. Detailed peripheral blood film examination helps determine the most probable causes of anemia. Presence of anisopoikilocytosis, nucleated RBCs, polychromatophils, abnormal leukocyte, giant platelets, and $\mathrm{Rbc}$ morphology provide important diagnostic clues. Bone marrow examination is an important diagnostic tool in hematology which helps to evaluate various causes of pancytopenia. Combined deficiency anemia was the commonest cause of pancytopenia in this study which indicates the high prevalence of nutritional anemia in our region. In patient presenting with pancytopenia and hepatosplenomegaly, bone marrow aspiration plays an essential role in diagnosing smear negative but marrow positive plasmodium infection and ruling out myelodysplastic syndrome.

The present study concludes that detailed physical examination, primary hematological investigations along with bone marrow examination is helpful in diagnosis and to rule out the various etiology of pancytopenia and thus it helps in further management of the patient.

\section{REFERENCES}

1. Sharma N, Bhatia PK, Kaul KK, Sharma S, Sharma M. A clinico-hematological study of pancytopenia: An experience of a tertiary care teaching hospital, Jammu, India. IJPO. 2017;4(4):632-637.

2. Basha M, Aziz M, Ali SM, Alam K, Alam F, Ahmed M: Evaluation of hematological parameters and bone marrow in Indian patients suffering from pancytopenia. BJMHR. 2016;3(4):1-15.

3. Khodke K, Marwah S, Buxi G, Yadav RB, Chaturvedi NK. Bone marrow examination in cases of pancytopenia. JIACM. 2001 Jan-June. 200; 2:1-2.

4. Dagdia KS, Deshmukh AT, Soni RR, Jane DS. Haematological indices and bone marrow morphology in pancytopenia/ bicytopnia. Egyptian J hematol 2015;41:23-26.

5. Javalgi AP, Dombale VD. Clinico-hematological analysis of pancytopenia: A bone marrow study. Natl J Lab Med. 2013;2(4):12-17.

6. Pathak R, Jha A, Sayami G. Evaluation of bone marrow in patients with pancytopenia. J Path Nepal. 2012 Jan 1;2(4):265-271.

7 Desalphine M, Bagga PK, Gupta PK, Kataria AS. To evaluate the role of bone marrow aspiration and bone marrow biopsy in pancytopenia. J Clin Diagn Res: JCDR. 2014 Nov;8(11): 11-15.

8. Krishna C, Haradanhalli RS. Epidemiological trends of malaria in an endemic district Tumkur, Karnataka. Int J Community Med Public Health.2017. Jun;4(6):2141-2145. 